\title{
Breast cancer clinicopathological presentation, gravity and challenges in Eritrea, East Africa: Management practice in a resource-poor setting
}

A Tesfamariam, ${ }^{1} \mathrm{MD} ; \mathrm{A}$ Gebremichael, ${ }^{1} \mathrm{PhD} ; \mathrm{J}$ Mufunda, ${ }^{2} \mathrm{PhD}$

${ }^{1}$ Orotta School of Medicine and Dentistry, Asmara, Eritrea

${ }^{2}$ World Health Organization, Lesotho

Corresponding author: A Tesfamariam (asmeromp@gmail.com)

Background. In Africa, breast cancer closely compares with cervical cancer as the most common malignancy affecting women and the incidence rates appear to be rising. Early detection of breast cancer is a key strategy for a good treatment outcome. However, there is no established protocol or guideline for management of breast cancer in Eritrea, East Africa.

Objective. To assess the clinicopathological presentation, gravity and management challenges presented in breast cancer treatment in Eritrea. Methods. Our investigation was a retrospective, descriptive study to assess the clinical features and severity of breast cancer at time of presentation. We reviewed the medical records of all patients who presented with breast malignancies over the 2-year period from 1 January 2007 to 31 December 2008. Results. Eighty-two patients ranging in age from 26 - 80 years (mean 48 years) were included in the study. Of these $51 \%$ were premenopausal women; $61 \%$ of the patients presented with breast mass only and the remainder with manifestations of local (mass plus discharge, breast pain or breast ulceration) or distant metastatic disease. More than $60 \%$ of the patients presented after $>2$ years following onset of symptoms. Two-thirds of patients had late stage (III or IV) disease. All except one case was managed surgically.

Conclusion. Most cases presented at younger age and advanced stage. These findings call for strengthening health education to promote early health-seeking behaviour and advocacy for the introduction of national screening, implementation of a management protocol and establishment of a radio-chemotherapy centre.

S Afr Med J 2013;103(8):526-528. DOI:10.7196/SAMJ.6829 


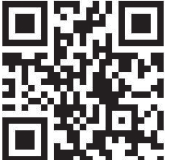

The reported incidence of breast cancer in sub-Saharan Africa is lower at 20 cases per 100000 population, than $90 / 100000$ in the West. ${ }^{[1]}$ In Africa, breast cancer closely compares with cervical cancer as the most common malignancy affecting women and the incidence rates appear to be rising. ${ }^{[2]}$ In Nigeria, for example, the incidence rate increased from $13.8-15.3 / 100000$ in the 1980 s to $33.6 / 100000$ in 1992 and 116/100 000 in 2001..$^{[3]}$

While mortality rates are declining in the developed world because of early diagnosis, screening and improved cancer treatment programmes, the converse is true in the developing world and in eastern and central Europe. ${ }^{[1,4]}$

There is a marked difference in distribution, presentation and outcome between developed and developing countries. ${ }^{[2,4]}$ In the USA, the percentage of patients presenting with stage I disease increased from $42.5 \%$ (1985) to $56.2 \%$ (1995), while the percentage of patients presenting with stage III or stage IV decreased from $18.3 \%$ to $11.6 \%$ during the same time period. ${ }^{[5]}$ In contrast, studies of women with breast cancer from Tunisia, ${ }^{[6]}$ Sudan, ${ }^{[7]}$ Nigeria ${ }^{[8]}$ and South Africa ${ }^{[9]}$ have shown that most patients present with advanced stages (III and IV) at first healthcare consultation. The geographic disparities in incidence rates can be attributed mainly to disease-reporting patterns and disease awareness.

About $70-90 \%$ of breast cancers present with breast masses and rarely with nipple discharge and pain. ${ }^{[10]}$ Triple Assessment clinical examination, imaging, cytology or tissue pathology of a biopsy specimen establishes the diagnosis for most women with suspected breast cancer. ${ }^{[1,12]}$

The prognosis, choice of treatment and outcome depend on accurate morphologic diagnosis, staging, histologic grade, hormone receptor status and identification of biomarkers. The latter two investigations are rarely possible in resource-poor countries. Treatment of early breast cancer is, however, less resource-intensive than advanced breast cancer with superior outcomes, the 5-year breast cancer survival rate ranges from $98 \%$ for stage I cancer to approximately $16 \%$ for stage IV cancer. ${ }^{[13]}$

According to previous studies undertaken in Eritrea, breast cancer is common in young women and there is regional variation. ${ }^{[14,15]}$ Information regarding clinicopathological presentation, gravity and the diagnostic and treatment approach is limited and has not been well documented. Moreover, there is no established protocol/guideline for management of breast cancer in Eritrea.

\section{Objective}

To assess (i) the clinicopathological presentation, (ii) severity of breast cancer at presentation, (iii) the methods followed in establishing the diagnosis, and (iv) the subsequent management of patients at 3 referral hospitals in Eritrea, East Africa.

\section{Methods \\ Study design}

Our investigation was a retrospective, descriptive study of breast cancer in patients who attended the General Surgical Units of 3 hospitals - Orotta Medical Surgical National Referral Hospital, Halibet Hospital and Sembel Hospital - from 1 January 2007 to 31 December 2008. These hospitals serve more than three-quarters of the Eritrean population requiring surgical interventions.

\section{Study population and data collection}

We reviewed 90 medical records over the 2-year period, of which we excluded 8 samples where data (age, sex, duration of symptoms, radiological, pathology, and operative reports and treatment received) were incomplete. Demographic details, clinical presentation and duration of symptoms at initial diagnosis, radiological data, cytological and histological findings, treatment and postoperative data of all patients were entered into our study protocol. Checklists for appropriate management, according to the recommended guideline for lowincome countries, ${ }^{[16]}$ were prepared during data collection. The WHO Tumour, Node and Metastasis (TNM) tumour classification and cancer staging system was used in this study. ${ }^{[17]}$ We performed descriptive data analysis using SPSS software (version 14).

\section{Results}

Of the 82 patients in our study, women constituted 94\% (female:male ratio 15:1.). The age range was 26 - 80 years (mean 48.46 years; standard deviation (SD) 16.2), the majority of patients were $\leq 55$ years with $10 \%>75$ years.

Seventy-eight (95\%) patients were married and $4(5 \%)$ were single. Geographically, 51 (62.2\%) patients were from the Central Zone,
$17(20.7 \%)$ from the South zone, 5 (6.1\%) from the Gash-Barka region, 4 (4.9\%) from the Southern Red Sea zone and the remaining $3.7 \%$ and $2.4 \%$ from the Northern Red Sea and Anseba zones, respectively.

The majority of patients presented with breast mass only (61\%), followed by breast mass plus discharge, pain or ulceration (36.6\%) and the remainder with breast pain only (2.4\%) with a left-side predominance of disease (56\%). The duration from onset of symptoms to first presentation ranged from 3 months to 10 years (mean 2.9 years; SEM 0.27) (Table 1).

Radiologic imaging (mammography/ ultrasonography or both) was undertaken with only $29 \%$ of patients; $73 \%$ underwent cytological examination of their breast masses prior to histological diagnosis. One-third of patients had resorted to traditional medicine as their first choice of treatment. Infiltrative ductal carcinoma accounted for $82 \%$ of all cancers. Invasive lobular carcinoma, papillary carcinoma and recurrence each accounted for $1.2 \%$. Cribriform, medullary carcinoma and phyllodes with other non-specified malignancies accounted for $2.4 \%, 4.0 \%$ and $8.3 \%$; respectively. More than two-thirds of the cases were of advanced stage (III and IV, 46\% and $18 \%$; respectively) (Table 1 ).

Three-quarters of patients had positive lymph node involvement and $18 \%$ had distant organ metastasis, chiefly to the lungs and liver, with associated symptoms of cough and jaundice, respectively (Table 2).

The hormone receptor status and biomarker profile had not been established in any cases. Treatment modalities ranged from excisional lumpectomy to palliative wound clearance: excisional lumpectomy $(n=5 ; 6.1 \%)$ (the tumour-free margin could not be defined intraoperatively in all these cases), modified radical mastectomy (MRM) ( $n=34 ; 41.5 \%)$, radical mastectomy $(n=6 ; 7.3 \%)$, radical mastectomy with axillary lymph node dissection $(n=26$; $31.7 \%)$ and wound debridement ( $n=9 ; 11 \%)$. Only 1 patient had MRM and chemotherapy. One patient refused mastectomy.

Of 7 patients with stage I disease, 4 underwent excisional lumpectomy, 2 had

Table 1. Cancer stage distribution v. duration of symptoms at first presentation

\begin{tabular}{clllll}
\hline & \multicolumn{4}{c}{ Duration of symptoms (months), $\boldsymbol{n}$} & \multirow{2}{*}{ Total } \\
\cline { 2 - 5 } Stage & $\mathbf{3 - 1 2}$ & $\mathbf{1 3 - 4 8}$ & $\mathbf{4 9 - 8 4}$ & $\mathbf{7 5 - 2 4 0}$ & $\mathbf{N}(\%)$ \\
\hline I & 4 & 2 & 0 & 1 & $7(9)$ \\
II & 14 & 7 & 1 & 0 & $22(27)$ \\
III & 11 & 18 & 9 & 0 & $38(46)$ \\
IV & 1 & 3 & 6 & 5 & $15(18)$ \\
Total, $N$ & 30 & 30 & 16 & 6 & 82
\end{tabular}


Table 2. Distribution of patients with distant organ/system metastasis and grave signs $(N=82)$

\begin{tabular}{lll}
\hline & Cases, $\boldsymbol{n}$ & Clinical presentation \\
\hline Distant organ/system metastasis & & \\
Vertebral bone & 1 & Breast mass and back pain \\
Vertebral bone and brain & 1 & Breast mass, back pain and hemiplegia \\
Vertebral bone and liver & 1 & Breast mass, back pain and jaundice \\
Liver & 4 & Breast mass and jaundice \\
Liver and lung & 2 & Breast mass, jaundice and cough \\
Lung & 6 & Breast mass and cough \\
No distant metastasis & 67 & Breast mass, pain and discharge
\end{tabular}

MRM and 1 refused surgical intervention. Of 22 patients with stage II disease, 18 underwent MRM, 3 radical mastectomy and 1 MRM with axillary lymph node dissection. Stage IV cancer patients received palliative intervention and only 6/15 underwent radical mastectomy with axillary lymph node dissection.

\section{Discussion}

In Eritrea and other East African countries, cancer is a neglected disease. Information on cancer is limited by the lack of a cancer registry and local evidence-based research.

In our study, $83 \%$ of the cases were from the highlands (the Central and South zones), which are densely populated and where there is better access to healthcare facilities and better health-seeking behaviour than the lowlands. This geographic disparity between the highlands and lowlands and between urban and rural populaces is consistent with the literature, which has documented reasons for these disparities..$^{[1,4,8]}$ Our study cannot completely reflect the incidence and prevalence of breast cancer in Eritrea because it was carried out at tertiary hospitals that attend to only $75 \%$ of the population.

In developed countries, breast cancer is a disease of older women. More than 50\% of patients in our study were premenopausal, similar to reports from Nigeria and other developing countries. ${ }^{[4,7,8,15]}$ The incidence of breast cancer typically increases with age and it is rare in patients $<25$ years old. In the white population the incidence is $<0.5 \%$ in patients $<35$ years old. ${ }^{[4]}$ In our study $26.8 \%$ of cases were $<35$ years old. Thus, our results indicate that in Eritrea breast cancer is a disease of younger, mainly premenopausal women.

In developing and resource-poor countries women with breast cancer present very late in the course of the disease, ${ }^{[7,8,18]}$ despite symptoms being easily identifiable during routine self-examination.

In our study $>70 \%$ of patients sought consultation $>1$ year after noticing symptoms and presented with advanced stage disease;
97\% presented with breast mass with or without additional symptoms such as pain,

The proportion of patients resorting to traditional therapy (e.g. herbal, holy water, soil) indicated that women in our society have poor attitudes towards western medicine, further exacerbating the poor outcome and prognosis of the disease. None presented with ductal carcinoma in situ, with $91 \%$ having infiltrating/invasive ductal carcinomas with a variety of subhistological types consistent with the literature from developing countries ${ }^{[4,6-8,17,18]}$ where invasive ductal carcinoma is reported in $70-85 \%$ of cases. ${ }^{[17]}$

Consistent with a study from Nigeria, ${ }^{[8]}$ $18 \%$ of patients had distant organ metastasis to the liver, lung, vertebrae and brain.

Breast cancer is a heterogeneous disease. Treatment outcomes depend on early and accurate diagnosis, the pathology of the disease, and the availability of adequate resources. While Triple Assessment is the gold standard for evaluation of breast cancer and has a specificity of diagnosis approaching $100 \%,{ }^{[11,12]}$ this was undertaken in only $24 \%$ of our cases. Hormone receptor analysis is not available in Eritrea.

The only treatment available in our setting is surgery, which in our patients ranged from excisional lumpectomy to radical mastectomy with axillary lymph node dissection. While surgery in combination with radiation therapy is the management of choice for early breast malignancies, and hormonal therapy and chemotherapy are administered to advanced cases where systemic metastasis is presumed, ${ }^{[16]}$ this is not established in Eritrea. An additional challenge is the lack of frozen-section procedure for the assessment of tumour-free margins, with the result that breast conservation therapy is hardly possible.

Without the right techniques to identify cancer-free margins, the risk of recurrence cannot be minimised. Furthermore, a radiochemotherapy centre for cancer has yet to be established in Eritrea. discharge and ulceration.

\section{Conclusions}

Our study, using retrospective and hospitalbased data, shows that breast cancer patients presented at a younger age, with advanced disease.

\section{Limitations of the study}

This retrospective case series based on hospital data from medical records of patients had a relatively small sample size and the true distribution of the disease in the country might not, therefore, be reflected.

Acknowledgments. Our appreciation goes to $\mathrm{Mr}$ Kibrom Hailu for inspiration and his dedication in data collection. We are grateful to Dr T Chinyanga for editorial support. We express our gratitude to the staff of the Histopathology Department, the 3 hospitals and the card archives for providing us with valuable information. Finally, AT is grateful to his family, especially his mother, for their endless encouragement in writing this paper.

\section{References}

1. Fregene A, Newman LA. Breast cancer in cub-Saharan Africa: How does it relate to breast cancer in African-American women? Cancer 2005;103(8):1540-1550. [http://dx.doi.org/10.1002/cncr.20978]

2. Ferlay J, Bray F, Pisani P, Parkin DM. Cancer Incidence, Mortality and Prevalence Worldwide. France: IARC Press, 2004.

3. Adebamowo CA, Ajayi OO. Breast cancer in Nigeria. West Afr J Med 2000;19(3):179-191.

4. Bhikoo R, Srinivasa S, Yu T-Z, Moss D, Hill AG. Systematic review of breast cancer biology in developing countries (Part 1): Africa, the Middle East, Eastern Europe, Mexico, the Caribbean and South America. Cancers 2011;3(2):2358-2381. [http://dx.doi.org/10.3390/ cancers3022358]

5. Abrams JS. Adjuvant therapy for breast cancer - results from the USA consensus conference. Breast Cancer; 2001;8(4):298-304.

6. Ben Ahmed S, Aloulou S, Bibi M, et al. Breast cancer prognosis in Tunisian women: Analysis of a hospital series of 729 patients. Sante Publique 2002;14(3):231-241.

7. Elgaili EM, Abuidris DO, Rahman M, Michalek AM, Mohammed SI. Breast cancer burden in central Sudan. Int J Womens Health S1. Breast cancer burden in central Sudan. Int I Wom

8. Adesunkanmi ARK, Lawal OO, Adelusola KA, Durosimi MA. The severity, outcome, and challenge of breast cancer in Nigeria. Breast 2006;15(3):399-409. [http://dx.doi.org/10.1016/j.breast.2005.06.008] 9. Vorobiof DA, Sitas F, Vorobiof G. Breast cancer incidence in South Africa. J Clin Oncol 2001;19:125-127.

10. Kumar V, Abbas AK, Fausto N. Robbins and Cotran Pathologic Basis of Disease. 7th ed. Philadelphia: Elsevier Saunders, 2005:1137-1148.

11. Morris KT, Vetto JT, Petty JK, et al. A new score for the evaluation of palpable breast masses in women under age 40 . Am J Surg 2002;184(4):346-347. [http://dx.doi.org/10.1016/ S0002-9610(02)00947-9]

12. Klein S. Evaluation of breast masses. Am Fam Physician 2005;71(9):1731-1739.

13. Shenkier T, Weir L, Levine M, et al. Clinical practice guidelines for the care and treatment of breast cancer: 15. Treatment for women with stage III or locally advanced breast cancer. CMAJ 2004;170(6):983-994. [http://dx.doi.org/10.1503/cmaj.1030944]

14. Ghebrehiwet M, Paulos E, Andeberhan T. The role of combined
2004:170(6):93-994. http://dx.doi.org/10.1503/cmaj.103094] 14. Ghebrehiwet M, Paulos E, Andeberhan T. The role of combined
ultrasonography and mammography in the diagnosis of breast cancer in Eritrean women with palpable abnormalities of the breast. J Eritrean Med Assoc 2007;2(1):2-7.

15. Tesfamariam A, Parilla F, Paulos E, Mufunda J, Gebremichael A. Clinicohistopathological evaluation of breast masses and profile of breast diseases in Eritrea: A case of poor concordance between clinical and histological diagnosis. J Eritrean Med Assoc 2008;3(1):32-35.

16. Anderson BO, Yip C-H, Smith R A, et al. Guideline implementation for breast healthcare in low-income and middleincome countries. Cancer 2008;113(S8):2221-2243. [http://dx.doi. income countries. Cance
$\mathrm{org} / 10.1002 /$ cncr.23844]

17. Tavassoéli FA, Devilee P. World Health Organization Classification of Tumours. Pathology and Genetics of Tumours of Classification of Tumours. Pathology and Genetics of Tumours of
the Breast and Female Genital Organs. $5^{\text {th }}$ ed. Lyon: IARC; 2003.

the Breast and Female Genital Organs. $5^{\text {th }}$ ed. Lyon: IARC; 2003.
18. Hisham AN, Yip CH. Overview of breast cancer in Malaysian women: A problem with late diagnosis. Asian I Surg 2004; 27(2):130-133. [http://dx.doi.org/10.1016/S1015-9584(09)60326-2]

Accepted 26 April 2013. 